\title{
A VARIATIONAL MULTIPHASE LEVEL SET APPROACH TO SIMULTANEOUS SEGMENTATION AND BIAS CORRECTION
}

\author{
Kaihua Zhang ${ }^{a}$, Lei Zhang ${ }^{a, 1}$ and Su Zhang $^{b}$ \\ ${ }^{a}$ Dept. of Computing, The Hong Kong Polytechnic University, Hong Kong \\ ${ }^{b}$ Dept. of Biomedical Engineering, Shanghai Jiaotong University
}

\begin{abstract}
This paper presents a novel level set approach to simultaneous tissue segmentation and bias correction of Magnetic Resonance Imaging (MRI) images. We first model the distribution of intensity belonging to each tissue as a Gaussian distribution with spatially varying mean and variance. Then a sliding window is used to transform the intensity domain to another domain, where the distribution overlap between different tissues is significantly suppressed. A maximum likelihood objective function is defined for each point in the transformed domain, which is then integrated over the entire domain to form a variational level set formulation. Tissue segmentation and bias correction are simultaneously achieved via a level set evolution process. The proposed method is robust to initialization, thereby allowing automatic applications. Experiments on images of various modalities demonstrated the superior performance of the proposed approach over state-of-the-art methods.
\end{abstract}

Index Terms - level set, segmentation, bias field, variational method, energy minimization

\section{INTRODUCTION}

The intensity inhomogeneity often exists in magnetic resonance imaging (MRI) images due to the imperfection of imaging devices. The intensity inhomogeneity can be generally modeled as a smooth and spatially varying field, multiplied by the constant true signal of the same tissue in the measured image. This spatially varying field is named as the bias field. Bias correction is a procedure to estimate the bias field and restore the true signals, thereby eliminating the side effect of the intensity inhomogeneity [2][3]. Among various bias correction methods, those based on segmentation are most attractive. The parametric model based on the maximum-likelihood (ML) or maximum a posterior (MAP) probability is often used to unify segmentation and bias correction [2], whose parameters can be estimated by the expectation maximization (EM) algorithm [3][4]. However, such algorithms are sensitive to the initialization of the variables [1][3], which limits their applications in automatic segmentation.

Recently, Li et al. [2] proposed a variational level set approach to simultaneous segmentation and bias correction, which has many advantages such as robustness to initialization and good approximation to bias fields of general profiles. Their method is motivated by the weighted $\mathrm{K}$-means clustering, and we name it as weighted K-means variational level set (WKVLS) method. However, as we will see from the following discussions, the WKVLS method can be viewed as a special case of our proposed SVMLS (statistical and variational multiphase level set) method, while the latter is more accurate.

In this paper, we first define a maximum likelihood objective function for each point in a transformed domain, where the distribution overlap between different tissues can be suppressed to some extent, and then an energy functional is defined by integrating the maximum likelihood function over the entire image domain. We then incorporate this energy functional into a multiphase level set formulation. Tissue segmentation and bias correction are obtained via a level set evolution process. A salient advantage of our method is that the smoothness of the computed bias field is ensured by the normalized convolution [5] without extra cost. Moreover, the evolution is less sensitive to the initialization, thus well suited for automatic applications.

\section{METHODOLOGY}

\subsection{Statistical Model of Images with Intensity Inhomogeneity}

We adopt the following commonly used model to describe images with intensity inhomogeneity [3]:

$$
I(\mathrm{x})=b(\mathrm{x}) J(\mathrm{x})+n(\mathrm{x}), \mathrm{x} \in \Omega
$$

where $I(\mathrm{x}): \Omega \rightarrow \mathrm{R}$ is the measured image; $b(\mathrm{x}): \Omega \rightarrow \mathrm{R}$ is the bias field which is spatially variant; $J(\mathrm{x}): \Omega \rightarrow \mathrm{R}$ is the true signal which is assumed to be piecewise constant, i.e. $J(\mathrm{x})=\mathrm{c}_{i}$ for $\mathrm{x} \in \Omega_{i}$, where $\mathrm{c}_{i}$ is a constant; and $\Omega_{i}$ denotes the $i^{\text {th }}$ object domain, $i=1, \ldots, N$, where $N$ is the total number of object domains. Noise $n(\mathrm{x})$ is assumed to be Gaussian distributed with zero mean and variance $\sigma^{2}$ [3]. Thus the

\footnotetext{
${ }^{1}$ Corresponding author: cslzhang@comp.polyu.edu.hk.
} 
image intensity can be approximated by Gaussian distribution with mean $b J$ and variance $\sigma^{2}$. However, using only a single Gaussian model is not accurate enough to describe the statistical characteristics of the image intensity. In order to accurately model the intensity distribution, the intensity distribution in each object domain should be ascribed to a Gaussian model. The intensity corresponding to the object domain $\Omega_{i}$ is modeled by [6]

$$
p\left(I(\mathrm{y}) \mid \alpha_{i}\right)=\frac{1}{\sqrt{2 \pi} \sigma_{i}} \exp \left(-\frac{\left(I(\mathrm{y})-b(\mathrm{x}) c_{i}\right)^{2}}{2 \sigma_{i}^{2}}\right), \mathrm{y} \in \Omega_{i}
$$

where $\alpha_{i}=\left\{b, c_{i}, \sigma_{i}\right\} ; \sigma_{i}$ is a constant corresponding to the standard deviation of intensity in domain $\Omega_{i \text {. }}$ and $b(\mathrm{x}) \mathrm{c}_{i}$ is the local mean that is spatially varying.

\subsection{Energy Functional Formulation}

For each position $\mathrm{x}$ in the image domain $\Omega$, we consider a circular neighborhood center on it, i.e., $O_{\mathrm{x}}=\{\mathrm{y} \mid\|\mathrm{y}-\mathrm{x}\| \leq \rho\}$, where $\rho$ is the radius of the region $O_{\mathrm{x}}$. We define a mapping $T: I\left(\mathrm{x} \mid \alpha_{i}\right) \rightarrow \bar{I}\left(\mathrm{x} \mid \alpha_{i}\right)$ from the original image intensity domain $\mathscr{\mathscr { C }}(T)$ to another domain $\mathscr{R}(T)$ as follows

$$
\bar{I}\left(\mathrm{x} \mid \alpha_{i}\right)=\frac{1}{m_{i}(\mathrm{x})} \sum_{\mathrm{y} \in \Omega_{i} \cap O_{\mathrm{x}}} I\left(\mathrm{y} \mid \alpha_{i}\right)
$$

where $m_{i}(\mathrm{x})=\left\|\Omega_{i} \cap O_{\mathrm{x}}\right\|$. Since the intensity of pixel y can be assumed to be independently distributed [10], $\bar{I}\left(\mathrm{x} \mid \alpha_{i}\right)$ is also normal distributed with mean $b c_{i}$ and variance $\frac{\sigma_{i}^{2}}{m_{i}(\mathrm{x})}$. We can intuitively observe from Fig.1 that the overlap between adjacent regions can be suppressed (see the red dashed curves).

Since the intensity inhomogeneity varies smoothly across the entire image domain [3], we have the approximation $I\left(\mathrm{y} \mid \alpha_{i}\right) \approx I\left(\mathrm{x} \mid \alpha_{i}\right), \forall \mathrm{y} \in \Omega_{i} \cap O_{\mathrm{x}}$. Because the product of Gaussian probability density functions (PDF) is still Gaussian, we have

$$
\prod_{\mathrm{y} \in \Omega_{i} \cap O_{\mathrm{x}}} p\left(I\left(\mathrm{y} \mid \alpha_{i}\right)\right) \approx p\left(I\left(\mathrm{x} \mid \alpha_{i}\right)\right)^{m_{i}(\mathrm{x})} \propto N\left(b c_{i}, \frac{\sigma_{i}^{2}}{m_{i}(\mathrm{x})}\right)
$$

Let $D=\left\{\bar{I}\left(\mathrm{x} \mid \alpha_{i}\right), i=1, \ldots, N\right\}$, we have the following likelihood function

$$
p(D \mid \alpha)=\prod_{i=1}^{N} p\left(\bar{I}\left(\mathrm{x} \mid \alpha_{i}\right)\right) \propto \prod_{i=1}^{N} \prod_{\mathrm{y} \in \Omega_{i} \cap O_{\mathrm{x}}} p\left(I\left(\mathrm{y} \mid \alpha_{i}\right)\right)
$$

where $\alpha=\left\{\alpha_{i}, i=1, \ldots, N\right\}$.

We integrate this likelihood function over the entire image domain to define the following energy functional

$$
\begin{aligned}
E(\alpha) & \triangleq-\int_{\Omega} \log p(D \mid \alpha) \mathrm{dx} \\
& =\text { constant }-\sum_{i=1}^{N} \int_{\Omega} \int_{\Omega_{i} \cap O_{\mathrm{x}}} \log \left(p\left(I\left(\mathrm{y} \mid \alpha_{i}\right)\right)\right) \mathrm{dydx}
\end{aligned}
$$

Let $\mathcal{K}_{\rho}(\mathrm{x}, \mathrm{y})$ be the characteristic function of region $O_{\mathrm{x}}$

$$
\mathcal{K}_{\rho}(\mathrm{x}, \mathrm{y})=\left\{\begin{array}{l}
1,\|\mathrm{y}-\mathrm{x}\| \leq \rho \\
0, \text { else }
\end{array}\right.
$$

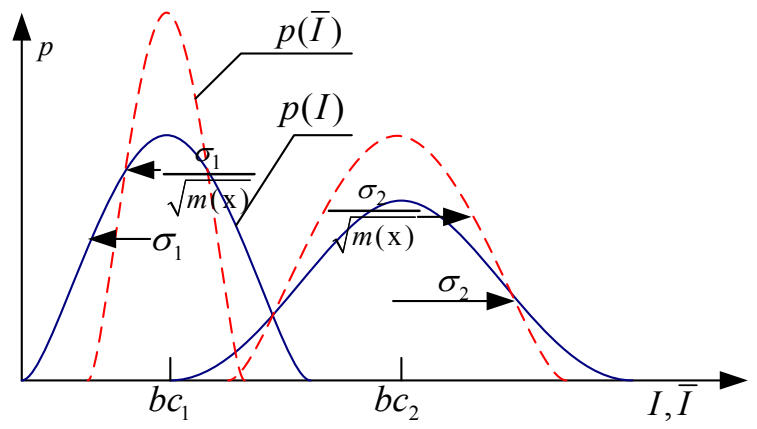

Fig. 1. Distributions of adjacent regions in the original image intensity domain (blue solid curves) and the transformed domain (red dashed curves).

With Eqs. (2) and (7), and eliminating the trivial constant term, $E(\alpha)$ can be re-written as

$$
E(\alpha)=\sum_{i=1}^{N} \int_{\Omega} \int_{\Omega_{i}} \mathcal{K}_{\rho}(\mathrm{x}, \mathrm{y})\left(\log \left(\sqrt{2 \pi} \sigma_{i}\right)+\frac{\left(I(\mathrm{y})-b(\mathrm{x}) c_{i}\right)^{2}}{2 \sigma_{i}^{2}}\right) \mathrm{dydx}
$$

In [2], Li et al proposed the weighted K-means variational level set (WKVLS) method. The energy functional of WKVLS is defined as follows

$$
E_{\alpha}^{\mathrm{WKVLS}}=\sum_{i=1}^{N} \int_{\Omega} \int_{\Omega_{i}} G_{\rho}(\mathrm{x}, \mathrm{y})\left(I(\mathrm{y})-b(\mathrm{x}) c_{i}\right)^{2} \mathrm{dydx}
$$

where $\alpha=\left\{b, c_{i}, i=1, \ldots, N\right\}$ and $G_{\rho}(\mathrm{x}, \mathrm{y})$ is a truncated Gaussian kernel where $G_{\rho}(\mathrm{x}, \mathrm{y})=0$ for $\|\mathrm{y}-\mathrm{x}\|>\rho$.

Obviously, if we set $\sigma_{i}$ in Eq. (8) to $1 / \sqrt{2 \pi}$, and $\mathcal{K}_{\rho}$ to a Gaussian kernel, $E(\alpha)$ in Eq. (6) and $E_{\alpha}^{\text {WKVLS }}$ in Eq. (9) will be the same except for some trivial constant. However, our model considers the variance differences among different tissues, which makes it more accurate than the WKVLS method.

\subsection{Level Set Formulation}

We use multiple level set functions (LSF) $\left\{\phi_{i}, i=1, \ldots, n\right\}$ to represent the regions $\left\{\Omega_{i}, i=1, \ldots, N\right\}$ with $N=2^{\text {n }}$ as in [8]. Let $M_{i}\left(\Phi_{N}(\cdot)\right)$ be the characteristic function of region $\Omega_{i}$, where $\Phi_{N}(\cdot)$ is a function of set $\left\{\phi_{i}, i=1, \ldots, n\right\}$. The energy functional $E(\alpha)$ in Eq. (6), which is called statistical and variational multiphase level set (SVMLS) in this paper, can be re-written as

$$
E_{\Phi_{N}, \alpha}^{\mathrm{SVMLS}}=\sum_{i=1}^{N} \int_{\Omega} d_{i}(\mathrm{y}) M_{i}\left(\Phi_{N}(\mathrm{y})\right) \mathrm{dy}
$$

where $d_{i}(\mathrm{y})=\int_{\Omega} \mathcal{K}_{\rho}(\mathrm{x}, \mathrm{y})\left(\log \left(\sqrt{2 \pi} \sigma_{i}\right)+\frac{\left(I(\mathrm{y})-b(\mathrm{x}) c_{i}\right)^{2}}{2 \sigma_{i}^{2}}\right) \mathrm{dx}$.

We only describe the energy minimization for fourphase case in this paper. (For two-phase case or other cases, the procedure is similar.) In the four-phase case, $M_{i}$ is defined as follows [8] 


$$
\left\{\begin{array}{l}
M_{1}=H\left(\phi_{1}\right) H\left(\phi_{2}\right), M_{2}=H\left(\phi_{1}\right)\left(1-H\left(\phi_{2}\right)\right) \\
M_{3}=\left(1-H\left(\phi_{1}\right)\right) H\left(\phi_{2}\right), M_{4}=\left(1-H\left(\phi_{1}\right)\right)\left(1-H\left(\phi_{2}\right)\right)
\end{array}\right.
$$

where $H(\phi)$ is the Heaviside function, and we often use the regularized version $H_{\varepsilon}(\phi)=\frac{1}{2}\left[1+\frac{2}{\pi} \arctan \left(\frac{\phi}{\varepsilon}\right)\right]$ in practice.

The minimizer of $E_{\Phi_{4}, \alpha}^{\mathrm{SVMLS}}$ in each variable is obtained by fixing other variables. The minimizers of the variables $c=\left\{c_{i}, i=1, \ldots, 4\right\}, b$ and $\sigma=\left\{\sigma_{i}, i=1, \ldots, 4\right\}$ are given as follows, respectively

$$
\left\{\begin{array}{l}
c_{i}=\frac{\int\left(\mathcal{K}_{\rho} * b\right) I M_{i}\left(\Phi_{4}\right) \mathrm{dy}}{\int\left(\mathcal{K}_{\rho} * b^{2}\right) M_{i}\left(\Phi_{4}\right) \mathrm{dy}}, b=\frac{\sum_{i=1}^{4} \mathcal{K}_{\rho} *\left(I M_{i}\left(\Phi_{4}\right)\right) \cdot \frac{c_{i}}{\sigma_{i}^{2}}}{\sum_{i=1}^{4} \mathcal{K}_{\rho} * M_{i}\left(\Phi_{4}\right) \cdot \frac{c_{i}^{2}}{\sigma_{i}^{2}}} \\
\sigma_{i}=\sqrt{\frac{\iint \mathcal{K}_{\rho}(\mathrm{y}, \mathrm{x})\left(I(\mathrm{y})-b(\mathrm{x}) c_{i}\right)^{2} M_{i}\left(\Phi_{4}(\mathrm{y})\right) \mathrm{dydx}}{\iint \mathcal{K}_{\rho}(\mathrm{y}, \mathrm{x}) M_{i}\left(\Phi_{4}(\mathrm{y})\right) \mathrm{dydx}}}
\end{array}\right.
$$

Obviously, the smoothness of the bias field $b$ is ensured by the normalized convolution [5], so it is general to images of various modalities.

Minimizing the energy functional $E_{\Phi_{4}, \alpha}^{\mathrm{SVMLS}}$ w.r.t $\phi_{1}$ and $\phi_{2}$, respectively, we derive the corresponding gradient descent as follows

$$
\left\{\begin{array}{l}
\frac{\partial \phi_{1}}{\partial t}=-\left[\left(d_{1}-d_{2}-d_{3}+d_{4}\right) H\left(\phi_{2}\right)+d_{2}-d_{4}\right] \delta\left(\phi_{1}\right) \\
\frac{\partial \phi_{2}}{\partial t}=-\left[\left(d_{1}-d_{2}-d_{3}+d_{4}\right) H\left(\phi_{1}\right)+d_{3}-d_{4}\right] \delta\left(\phi_{2}\right)
\end{array}\right.
$$

where $\delta(\phi)$ is the Dirac function. In practice, we often use the regularized version $\delta_{\varepsilon}(\phi)=\frac{1}{\pi} \cdot \frac{\varepsilon}{\varepsilon^{2}+\phi^{2}}$.

\subsection{Implementation}

Theorem1. A signed distance function (SDF) $\phi$ satisfies $|\nabla \phi|=1$ [7]. Conversely, any function $\phi$ satisfies $|\nabla \phi|=1$ is an SDF plus a constant [9].

Proposition1. An SDF $\phi$ convolving by a $\zeta \times \zeta$ normalized constant kernel $K_{\zeta}^{c}$ is an SDF plus a constant.

Proof: Let $\phi^{\text {new }}=\phi * K_{\zeta}^{c}$. There is

$$
\begin{aligned}
\left|\nabla \phi^{\text {new }}\right| & =\left|\nabla\left(\phi * K_{\zeta}^{c}\right)\right|=\left|(\nabla \phi) * K_{\zeta}^{c}\right|=\sqrt{\left(\phi_{x} * K_{\zeta}^{c}\right)^{2}+\left(\phi_{y} * K_{\zeta}^{c}\right)^{2}} \\
& =\sqrt{\phi_{x}^{2}+\phi_{y}^{2}} * K_{\zeta}^{c}=|\nabla \phi| * K_{\zeta}^{c} .
\end{aligned}
$$

For $|\nabla \phi|=1$ and $K_{\zeta}^{c} * 1=\int K_{\zeta}^{c} \mathrm{~d} \Omega=1$, we have $\left|\nabla \phi^{\text {new }}\right|=1$. So $\phi^{\text {new }}$ is an SDF plus a constant. End of proof.

The main steps of the algorithm can be summarized as

A. Initialize $\phi_{1}$ and $\phi_{2}$ to be SDFs or constants with different signs inside and outside contour, respectively.
B. Keep $\phi_{1}$ and $\phi_{2}$ fixed, optimize and update the variables $c, b$, and $\sigma$ by Eq. (12), respectively.

C. Keep $c, b$, and $\sigma$ fixed, evolve $\phi_{1}$ and $\phi_{2}$ according to Eq. (13), respectively.

D. Check whether the convergence has been reached. If not, return to $B$.

During the evolution process, the LSFs (level set function) need to be periodically regularized in order to keep numeric stability. In this paper, we regularize $\phi_{1}$ and $\phi_{2}$ by convolving a constant kernel (degenerated Gaussian kernel) after each iteration in step $C$, which can be seen as a special case of the method proposed in [7], while the later uses a Gaussian kernel. As Proposition 1 shows, with our approach, the LSF keeps the property of SDF, so it can make the evolution stable. The size of the kernel $\zeta \times \zeta$ should not be too large. In our experiments, we set $\zeta=3$ for efficiency. We also found that in step $\boldsymbol{A}$, it is reliable to simply initialize $\phi_{1}$ and $\phi_{2}$ to be constants with different signs inside and outside contour. With this initialization, the convergence speed is much faster than that by SDF, and the tissue segmentation results are also much more accurate.

\section{EXPERIMENTAL RESULTS}

We compare our method with the WKVLS method [2], which has better performance than the well-known methods proposed by Well et al. [4] and Leemput et al. [1]. We use the same initializations in both models for fair comparisons, and initialize $\left\{\sigma_{i}=1, i=1, \ldots, 4\right\}, \rho=4.5$ in our algorithm. The code of the proposed method can be downloaded at http://www4.comp.polyu.edu.hk/ cslzhang/code.htm.

Fig. 2 shows the result for a $3 \mathrm{~T}$ MRI image. There are four classes of tissues: whiter matter (WM), gray matter (GM), cerebrospinal fluid (CSF) and the background. As Fig. 2(d) shows, the histogram of the bias corrected image by our method has three well-separated peaks, which correspond to the background, GM, and WM from left to right, respectively, while the peak of the CSF (the one between the background and GM) is not distinct since its volume is relatively small. However, the histogram by the WKVLS has no obvious well-defined and well-separated peaks. The tissue segmentation result by our method (see Fig.2 (a)) is much more accurate than that by the WKVLS method (see Fig.2 (b)) (e.g. the regions that the red arrows point to). It should be noted that it is very flexible to initialize the LSFs in our method. The initial contours can be set inside, outside or across the object boundary. As shown in Fig.2 (a) and (c), the results are similar. To save space, we did not show the results of other initializations.

Fig. 3 shows the result on a 7T MR image, whose intensity inhomogeneity is so severe that even the experts are difficult to tell the structures. By applying our method, the image quality can be significantly improved. Many regions (e.g. the regions inside the red circles and ellipses) 
that are difficult to distinguish can be much better viewed after the bias correction.

\section{CONCLUSION}

This paper presents a novel statistical and variational multiphase level set (SVMLS) approach to simultaneous bias correction and tissue segmentation for MRI image. The smoothness of the bias field is intrinsically ensured by the normalized convolution without any extra costs, which makes our method well fitted for images of various modalities, such as 3T and 7T MRI images. Moreover, the proposed SVMLS algorithm is robust to the initializations, therefore allowing for fully automatic applications. Comparisons with state-of-the-art method on real MRI images show the advantages of the proposed algorithm.

\section{ACKNOWLEDGEMENT}

This research is supported by the Hong Kong RGC General Research Fund (PolyU 5375/09E), the National Basic Research Program of China (973 Program, No. 2010CB732506) and NSFC (No. 60972110).

\section{REFERENCES}

[1] K.Leemput, F.Maes, D.Vandermeulen, and P.Suetens. "Automated model-based bias field correction of MR
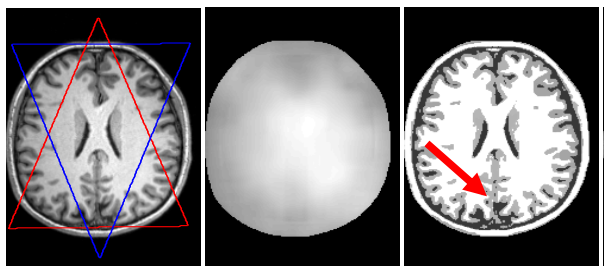

(a)
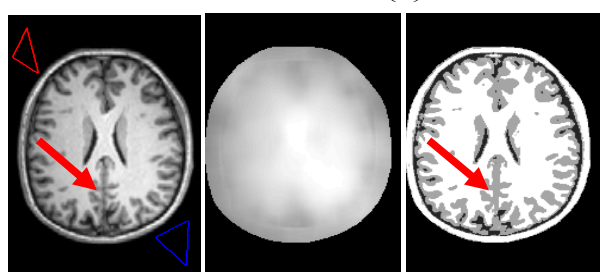

(c)

images of the brain", IEEE Trans.Med.Imag., vol.18, pp.885-896, Oct.1999.

[2] C.Li, R.Huang, Z.Ding, C.Gatenby, D.Metaxas, and J.Gore, "A variational level set approach to segmentation and bias correction of medical images with intensity inhomogeneity", MICCAI., vol. LNCS 5242, pp. 1083-1091, 2008.

[3] U.Vovk, F.Pernus, and B.Likar, " A review of methods for correction of intensity inhomogeneity in MRI", IEEE Trans.Med.Imag., vol.26, pp.405-421, Mar.2007.

[4] W.Wells, E.Grimson, R.Kikinis, and F.Jolesz, " Adaptive segmentation of MRI data", IEEE Trans. Med. Imag., vol.15, pp.429-442, 1996.

[5] H. Knutsson and C.-F. Westin,"Normalized and Differential Convolution: Methods for Interpolation and Filtering of Incomplete and Uncertain data", CVPR., pp.515-523,1993.

[6] T.Brox, "From pixels to regions: partial differential equations in image analysis", Ph.D. Thesis, Saarland University, Germany, 2005.

[7] K.Zhang, H.Song, L.Zhang, "Active contours driven by local image fitting energy", Pattern Recognition, 2010.

[8] Vese.L, Chan.T, "A multiphase level set framework for image segmentation using the mumford and shah model", $I J C V$., vol.50,pp.271-293, 2002.

[9] C. Li, C.Xu, C.Gui, M.Fox, "Level Set Evolution Without Re-initialization: A New Variational Formulation", CVPR., 2005.

[10] S. Zhu and A.Yuille, "Region competition: Unifying snakes, region growing, and bayes/mdl for multiband image segmentation", IEEE T-PAMI., vol.18, pp. 884-900, 1996.
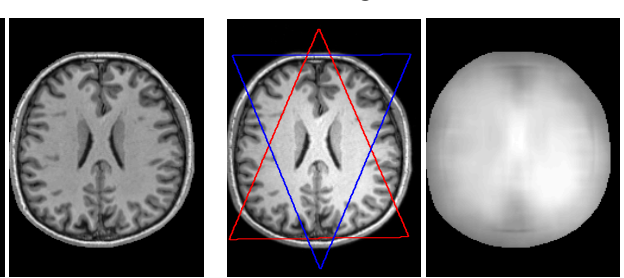

(b)
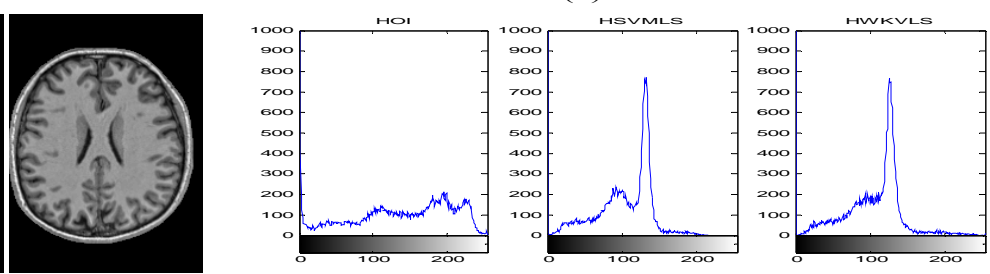

(d)

Fig. 2. (a) and (b): From left to right: the initializations of level set functions, estimated bias fields, tissue classification results, and bias corrected images by our method (SVMLS) and the WKVLS method, respectively. (c): An arbitrary initialization of level set functions and the corresponding experimental results. (d) Histograms of original image (HOI), bias corrected image by our method
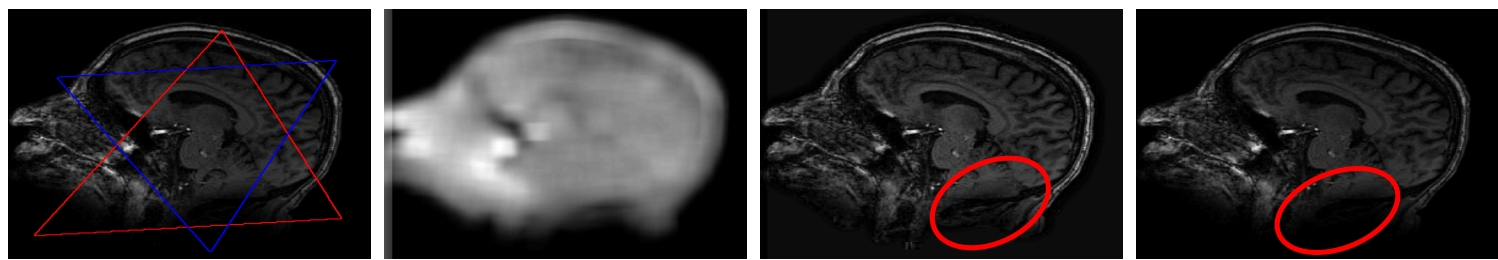

Fig.3. Experiments on 7T MR image. Column 1: Initial contours; Column 2: Estimated bias field. Column 3: Bias correction image. Column 4: Original image. 